\title{
BRÈVES
}

Ta Un déficit en une enzyme des lysosomes, la B mannosidase, avait été recherchée en vain chez des centaines de malades depuis la description en 1981 d'une $B$ mannosidose chez la chèvre. On vient d'en découvrir deux cas, l'un sur un enfant de trois ans aux USA, l'autre sur un adulte originaire de l'Inde. La relative bénignité des symptômes de ces premiers malades - retard intellectuel accompagné ou non de dysmorphie faciale - contraste avec l'extrême gravité de la maladie caprine.

Wenger DA, et al. $N$ Engl J Med 1986 ; 315 : 1201-5.

Cooper A, et al. Ibid, 1986 ; 315 : 1231.
- Un modèle de maladie de Huntington chez le rat par intoxication à l'acide quinolinique, métabolite du tryptophane, a été proposé $\left(\mathrm{m} / \mathrm{s} n^{\circ} 7\right.$, vol. 2, p. 400). Une équipe de Standford vient de montrer que les neurones qui, en culture, résistent au quinolinate ou à son agoniste, le N-méthyl-Daspartate, possèdent un marqueur spécifique, l'enzyme NADPH diaphorase, une enzyme d'oxydoréduction dont on ignore d'ailleurs le rôle physiologique. Or les neurones à NADPH diaphorase sont électivement épargnés dans le cerveau des sujets atteints de maladie de Huntington. C'est là un argument en faveur du rôle du quinolinate ou d'un toxique de même type d'action dans la genèse de la maladie.

Koh JY, Peters S, Choi DW. Science $1986 ; 233: 73-6$.
Lorsque des plaquettes adhèrent à une brèche vasculaire importante, le TGF (transforming growth factor, voir $\mathrm{m} / \mathrm{s} n \circ 8$, vol. 2, p. 467) qu'elles libèrent inhibe la prolifération des cellules endothéliales... et retarde donc la fermeture de cette brèche. Ceci pourrait permettre à d'autres substances plaquettaires d'atteindre les cellules musculaires lisses de la paroi vasculaire, et par conséquent de stimuler leur prolifération, indispensable à une reconstitution complète et solide de la paroi.

Heimark RL. Science $1986 ; 233$ 1070-80.

\section{La neuroleukine, à la fois lymphokine et facteur neurotrophique}

La présence de fibres musculaires dénervées dans un muscle induit la croissance de neurites à partir des axones innervant les fibres adjacentes. Ce processus est stimulé par une protéine de poids moléculaire 56000 , sécrétée par des cultures organotypiques de muscle dénervé. Cette protéine agit sur les motoneurones spinaux, certains neurones sensoriels, mais pas sur les neurones sympathiques et parasympathiques. En culture, elle permet la survie de neurones embryonnaires spinaux et de certains neurones sensoriels insensibles au $N G F$.

Comme cela est le cas pour EGF et $N G F\left(\mathrm{~m} / \mathrm{s} \mathrm{n}^{\circ}\right.$ 9, vol. 2, p. 527), le facteur neurotrophique ainsi identifié est également très abondant dans la glande sous-maxillaire de souris mâle à partir de laquelle il a pu être totalement purifié. L'ADN complémentaire de son messager a été cloné et sa séquence nucléotidique a été déterminée [1]. Celle$\mathrm{m} / \mathrm{s} n^{\circ} 2$ vol. 3, févria 87 ci a le potentiel de coder pour une protéine de 558 acides aminés (poids moléculaires: 62 803) qui a une homologie faible et partielle avec une région très conservée de la glycoprotéine d'enveloppe du virus du SIDA HIV. Le messager et la protéine sont largement distribués dans différents organes, mais sont particulièrement abondants dans le muscle squelettique.

De facon surprenante, le facteur neurotrophique est également une lymphokine sécrétée par des lymphocytes $T$ stimulés par des lectines (concanavaline $A$ ou phytohémagglutinine) et a donc été dénommé "neuroleukine" [2]. La neuroleukine produite par recombinaison génétique est capable, en présence de monocytes et de lymphocytes $T$, de stimuler les lymphocytes $B$, induisant la sécrétion polyclonale d'immunoglobulines $G$ sans provoquer de prolifération cellulaire. Elle est distincte des quelques lymphokines actives sur les lymphocy- tes $B$ connues jusqu'à présent, $B C G F$ ou $B C D F$ (B cell growth or differentiating factors).

La neuroleukine constitue donc un nouvel exemple des relations fonctionnelles entre le système immunitaire et le système nerveux $\left(\mathrm{m} / \mathrm{s} \mathrm{n}^{\circ} 9\right.$, vol. 2, p .401). De plus, l'homologie partielle observée avec la glycoprotéine de surface d'HIV peut suggérer que cette dernière serait capable de mimer certaines actions de la neuroleukine, expliquant ainsi l'activation polyclonale des lymphocytes $B$ avec déficit de l'immunité humorale et certains désordres neurologiques observés chez les malodes atteints de SIDA.

A. $\mathbf{K}$.

1. Gurney ME, Heinrich SP, Lee MR, Yin HS. Molecular cloning and expression of neuroleukin, a neurotrophic factor for spinal and sensory neurons. Science 1986 ; 234 : 566-74. 2. Gurney ME, Apatoff BR, Spear GT, et al. Neuroleukin : a lymphokine product of lectinstimulated T cells. Science $1986 ; 234: 574-81$. 
- Un réarrangement aberrant entre un segment $V_{H}$ codant pour la partie variable des chaînes lourdes d'immunoglobuline et les segments du gène $\alpha$ du récepteur T (TCR $\alpha)$ a été décrit, il y a quelques mois, dans un lymphome $T$ humain [1]. Le mécanisme en est l'inversion paracentrique du bras long du chromosome 14 amenant à contiguïté le segment $V_{H}$ (situé dans la région télomérique) et $\mathrm{J} \alpha$ (segment $\mathrm{J}$ du gène $\mathrm{TCR} \alpha$, situé initialement dans la région centromérique). Ce réarrangement aboutit à la formation d'un gène hybride fonctionnel, composé de segments des gènes d'immunoglobuline et du récepteur $T$. Un réarrangement semblable vient d'être démontré dans un lymphome B [2], semblant indiquer que la recombinaison au niveau des gènes d'immunoglobuline, aussi bien que du récepteur $T$, est possible tout à la fois dans les cellules $B$ et les cellules $T$, alors que la tendance actuelle était plutôt de considérer que l'accessibilité à la "recombinase " des gènes TCR était une caractéristique d'une cellule T, l'accessibilité des gènes d'immunoglobuline caractérisant les cellules B.

1. Baer R, et al. Cell 1985; 43 : 705-15.

2. Denny CT, et al. Science 1986 ; 234: 197-200.

L'infection par le virus du SIDA semble récente en Afrique comme dans les autres pays du monde. Avant 1975, il n'existait pas dans des sérums de malades d'Ouganda et d'autres pays africains d'anticorps anti HIV. Par conséquent, à ce jour il n'existe aucune preuve formelle que l'origine de l'épidémie mondiale actuelle soit africaine.

Levy JA, et al. Proc Natl Acad Sci
Les transcrits de l'ADN du virus de l'hépatite $B$ ont été étudiés sur des fragments de tissu hépatique obtenus par biopsie à l'aiguille. Lorsque l'ADN n'est pas intégré, le transcrit principal est l'antigène $\mathrm{HBc}$. Lorsque l'ADN est intégré au génome de l'hôte, seul l'antigène $\mathrm{HBs}$ est transcrit. La technique est plus sensible que la recherche de l'ADN lui-même pour identifier le virus $B$.

Yokosuka O, et al. $N$ Engl J Med 1986 ; 315 : 1187-92.

Les femmes à caryotype $\mathbf{X Y}$ ont fait l'objet de moins d'études que les hommes XX $(\mathrm{m} / \mathrm{s}$ $n^{\circ} 6$, vol. 1, p. 331 et $n^{\circ} 3$, vol. 2, p. 157). Même quand ce caryotype est pur, c'est-à-dire en l'absence d'une mosaïque avec des cellules $\mathrm{XO}$, il s'accompagne d'une dysgénésie gonadique et d'un syndrome de Turner. L'hypothèse mise en avant est celle d'une délétion du chromosome $\mathrm{Y}$, siégeant dans la région, encore non identifiée, qui détermine le sexe mâle, région localisée sur le bras court de l'Y. Deux cas portant une délétion cytogénétique de ce bras court étaient connus. Un pas de plus a été franchi par une équipe francoaméricaine qui a analysé deux nouveaux cas, dont la délétion est à peine reconnaissable au microscope, à l'aide de sondes d'ADN spécifiques du chromosome $\mathrm{Y}$. L'ADN de chacune de ces femmes porte des délétions, différentes entre elles, mais comportant une portion commune, délétions qui n'étaient pas présentes chez les pères. On peut espérer que l'emploi de sondes multiples spécifiques du chromosome $Y$ détectera chez certaines femmes XY des délétions qui échappent à l'examen cytogénétique. D'autre part ces méthodes permettront de cerner et peut-être d'identifier le locus déterminant du sexe masculin sur le chromosome $\mathrm{Y}$.

Disteche CM, et al. Proc Natl Acad Sci USA 1986 ; 83 : 7841-4.

Le syndrome du chromosome $\mathrm{X}$ fragile est une forme fréquente de rètard mental dont la transmission est récessive liée à l'X. Il arrive cependant que cette transmission "saute des générations ", des hommes apparemment sains étant alors transmetteurs. La fragilité du chromosome $\mathrm{X}$ entraîne des cassures en culture de cellule, localisées au niveau de la bande Xq27. La méthode des hybrides intersomatiques hommes-rongeurs permet de démontrer que, même chez les hommes normaux, un site fragile physiologique existe à ce niveau. Chez les hommes transmetteurs, un traitement des hybrides par la caféine permet de démontrer une fragilité du chromosome $\mathrm{X}$ intermédiaire entre celle des sujets normaux et des malades. La mutation responsable de la maladie entraîne donc une fragilité supplémentaire d'un site fragile physiologique et sa pénétrance est incomplète.

Ledbetter DH, et al. Nature 1986 ; 324 : 161-3.

La colchicine pourrait être utile pour traiter la cirrhose biliaire primitive. Chez 60 malades inclus dans un essai contrôlé, les tests biologiques hépatiques se sont améliorés et la mortalité au bout de deux ans était deux fois moins élevée dans le groupe traité que dans le groupe placebo. Toutefois, aucune amélioration histologique n'a été notée.

Kaplan MM, et al. $N$ Engl J Med 1986 ; 315 : 1448-54. 